\title{
Disorders in Amniotic Fluid
}

\section{Lynnae K Sauvage}

Professor, Department of Obstetrics and Gynecology, and Women's Health, John A Burns School of Medicine, University of Hawaii, Honolulu, Hawaii, USA

Correspondence: Lynnae K Sauvage

Department of Obstetrics and Gynecology, John A Burns School of Medicine

University of Hawaii, 1319 Punahou, 8th Floor, Honolulu, HI 96826

Tel: 808-235-5134, e-mail: mill8lynn@aol.com

\begin{abstract}
Disorders in amniotic fluid production occur frequently in pregnancy, and are associated with increased perinatal mortality and morbidity. This article will discuss amniotic fluid physiology, and will summarize noninvasive methods of measuring amniotic fluid. The definitions, common causes and possible therapeutic interventions available to treat oligohydramnios and polyhydramnios will be discussed.
\end{abstract}

Key words: Oligohydramnios, polyhydramnios, hydration, amnioinfusion.

\section{Learning objectives}

- To have an understanding of the mechanisms underlying the regulation of amniotic fluid volumes

- To understand the limitations of current clinical measurements of amniotic fluid volumes

- To know the definitions, causes and potential treatments of disorders in amniotic fluid.

\section{AMNIOTIC FLUID PHYSIOLOGY}

Disorders in amniotic fluid production occur frequently in pregnancy, and are associated with increased perinatal mortality and morbidity. ${ }^{1}$ An understanding of the mechanisms underlying the regulation of amniotic fluid is necessary to prevent or develop therapeutic interventions to treat disorders in amniotic fluid volumes. Four volume flows appear to affect fluid flux in the amniotic cavity: (i) fetal urine production, (ii) lung liquid secretion, (iii) fetal swallowing and (iv) intramembranous absorption. ${ }^{2}$ Fetal urine production, lung liquid production and fetal swallowing are regulated to meet fetal needs, and this can result in abnormalities in amniotic fluid volumes. Alternatively, intramembranous absorption may be responsible for regulation and maintenance of amniotic fluid volumes within the normal range. Intramembranous absorption of amniotic fluid has been predominately studied in the sheep model, as invasive techniques are required to quantify these fluid shifts and these studies cannot be performed in the human. The osmotic pressure gradient between amniotic fluid $(255 \mathrm{mosm} / \mathrm{kg})$ and fetal blood (180 mosm $/ \mathrm{kg}$ ) allows microscopic blood vessels in the ovine fetal membranes and placenta to absorb fluid from the amniotic cavity. Under normal conditions, approximately $250 \mathrm{ml}$ amniotic fluid per day leaves the ovine amniotic cavity and enters the fetal circulation. ${ }^{3}$ The injection of technetium- $99 \mathrm{~m}$ into the amniotic cavity of rhesus monkey fetuses with esophageal ligation results in a rapid absorption of Tc-99m into the fetal and maternal circulations, suggesting intramembranous absorption occurs in an animal model closely resembling the human. ${ }^{4}$ Studies in the ovine model are beginning to document the importance of intramembranous absorption in the regulation of amniotic fluid volumes. Water and arginine vasopressin (AVP) are absorbed into the fetal circulation more rapidly if the fetal esophagus is ligated than if it is intact, suggesting the rate of intramembranous absorption can change with the fetal condition. Additionally, infusion of large amounts of saline into the amniotic cavity of fetal sheep with esophageal ligation seldom results in polyhydramnios, suggesting large amounts of amniotic fluid can be absorbed through the blood vessels lining the placenta and membranes. ${ }^{5}$ Further research defining the mechanisms controlling intramembranous absorption, particularly in primates, will aid in the development of potential therapeutic interventions to regulate and normalize amniotic fluid volumes.

\section{MEASUREMENT OF AMNIOTIC FLUID}

Invasive techniques have accurately characterized amniotic fluid volume changes throughout gestation, ${ }^{6,7}$ but these techniques are not suitable for routine use in clinical practice. Ultrasound measurements are commonly performed; varying methods have been proposed including measurement of a single vertical pocket, ${ }^{8-10}$ a two-diameter pocket, ${ }^{2}$ or the four quadrant amniotic fluid index (AFI). ${ }^{11,12}$ In the ovine model, the AFI has a close linear relationship to actual amniotic fluid volume. ${ }^{12}$ Additionally, plots of AFI throughout human gestation are similar to those generated using invasive dye-dilution studies. Though the AFI is commonly utilized, and is the best of current 
clinical assessments of amniotic fluid volume, ${ }^{12}$ it remains inadequate to identify the extremes of amniotic fluid. The subjective assessment of amniotic fluid volumes is as accurate as an objective AFI measurement, and these assessments do not appear to be affected by operator experience. ${ }^{13} \mathrm{New}$ ultrasound technology is required with high, rapid, $3 \mathrm{D}$ resolution to accurately determine real-time amniotic fluid volumes.

\section{POLYHYDRAMNIOS}

Polyhydramnios is defined as an excessive amniotic fluid volume more than two standard deviations above the mean for a given gestational age. Accepted ultrasound criteria include a maximum vertical pocket of $>8 \mathrm{~cm}$ (Fig. 1), or an AFI of $>24 \mathrm{~cm} .{ }^{14}$ The incidence of polyhydramnios is approximately $1 \% .{ }^{15}$ Documentation of polyhydramnios is associated with an increased rate of maternal and fetal complications. ${ }^{16}$ Maternal complications include an increased rate of cesarean birth and placental abruption. Fetal complications include an increased rate of stillbirth, neonatal death, fetal distress, meconium-stained amniotic fluid, malpresentation, cord prolapse and macrosomia. About half of all cases of polyhydramnios are idiopathic, the rest are due to maternal diabetes or congenital malformation. When polyhydramnios is diagnosed during pregnancy, a diabetic screen and a thorough fetal ultrasound should be performed. In particular, the fetal size should be determined, the amniotic fluid quantified, the gastrointestinal tract should be imaged, and a biophysical profile should be performed to verify normal tone, movement and breathing. Absent or reduced fetal swallowing, due to gastrointestinal obstruction or neurologic abnormalities, can cause polyhydramnios. Gastrointestinal abnormalities can be functional (i.e. gastroschisis) or structural (i.e. cleft lip and palate, esophageal and intestinal atresias). ${ }^{2}$ Gastrointestinal obstruction can result in impaired fetal enteral nutrition, as the amniotic fluid contains fetal nutrients and growth factors, ultimately resulting in fetal growth restriction. ${ }^{2}$ Though some fetal anomalies (i.e. cleft lip, gastroschisis, and duodenal atresia (Fig. 2) are relatively easy to diagnose with prenatal ultrasound, some malformations (i.e. tracheal esophageal fistula, cleft palate, neurologic abnormalities) may not be diagnosed until after birth. Once polyhydramnios has been diagnosed, regular antepartum surveillance is indicated due to the increased risk of fetal distress and stillbirth.

Indomethacin has been utilized to treat severe or symptomatic polyhydramnios. This medication is efficacious and decreases amniotic fluid volumes in some (though not all) pregnancies complicated by the disorder. ${ }^{17}$ Administration of indomethacin decreases fetal urine production. ${ }^{18}$ Animal studies have shown indomethacin's suppression of prostaglandin production increases the release of AVP from the pituitary and enhances the efficacy of AVP in the fetal kidney. ${ }^{19}$ There are significant fetal side effects associated with maternal indomethacin administration. Fetal ductal constriction can occur,

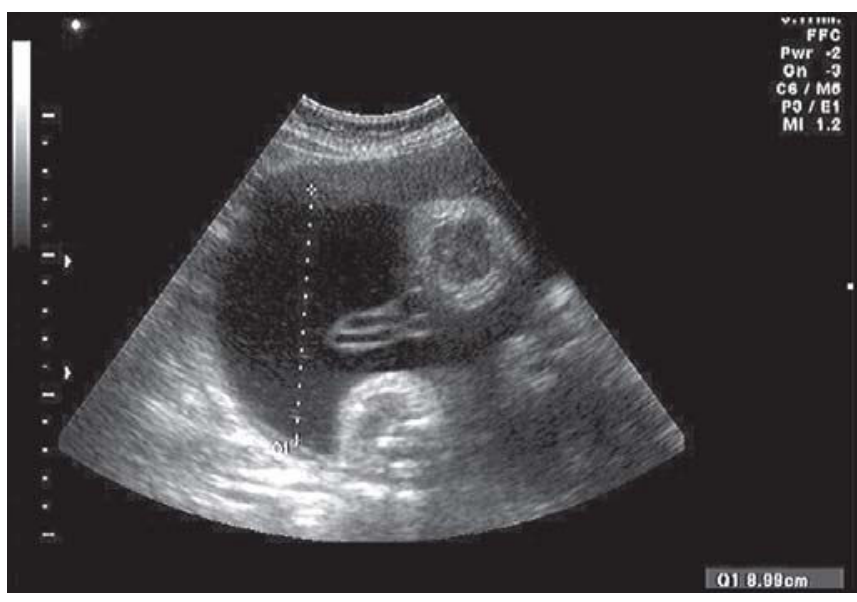

Fig. 1: Single vertical pocket amniotic fluid measurement $>8 \mathrm{~cm}$, consistent with polyhydramnios

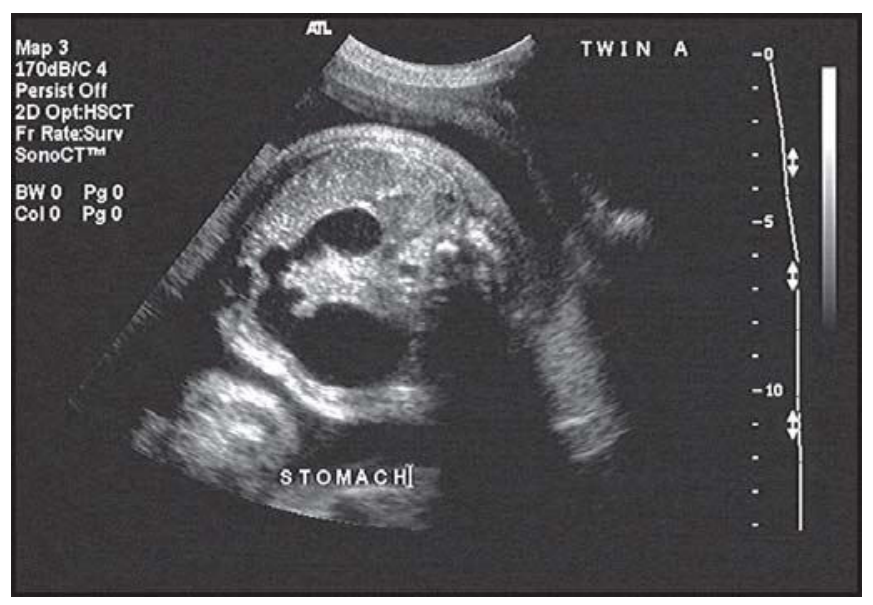

Fig. 2: Stomach "double bubble", consistent with duodenal stenosis

and the risk of ductal constriction increases as gestation advances, affecting approximately $10 \%$ of fetuses at 27 weeks of gestation and $50 \%$ of fetuses at 32 weeks of gestation. ${ }^{20}$ Thus this medication should be utilized conservatively with serial fetal echocardiograms and amniotic fluid measurements to monitor fetal wellbeing. Indomethacin should not be routinely utilized after 32 weeks gestation. Serial amnioreductions have also been utilized to treat symptomatic polyhydramnios.

\section{OLIGOHYDRAMNIOS}

Oligohydramnios is defined as a decreased amniotic fluid volume of $<318 \mathrm{ml}$ (as measured by dye-dilution studies) in late gestation. ${ }^{21}$ Accepted ultrasound criteria include an AFI $<5 \mathrm{~cm}$ and an AFI $<5$ th percentile for gestational age. ${ }^{2}$ Oligohydramnios occurs in $8.2 \%$ of antepartum patients, in $37.8 \%$ of laboring patients, and is associated with significantly increased 
perinatal morbidity and mortality. ${ }^{1,22}$ There are multiple causes of oligohydramnios, and management of the condition is individualized based on the maternal or fetal condition.

Regardless of gestational age, once oligohydramnios is diagnosed, preterm premature rupture of the fetal membranes must be excluded. In the first and second trimesters oligohydramnios can occur with fetal structural (i.e. fetal renal obstruction or renal agenesis) or chromosomal anomalies. ${ }^{2}$ Oligohydramnios diagnosed at this gestational age warrants careful fetal anatomical survey for evidence of fetal malformation or genetic syndrome, and possible invasive testing for fetal karyotype and viral infections. Oligohydramnios occurring early in gestation can compress the fetus and cause pulmonary hypoplasia and fetal deformation. This often results in stillbirth or neonatal death. Alternatively, oligohydramnios in the late second and third trimesters frequently occurs secondary to maternal medical disease (i.e. hypertensive diseases, antiphospholipid antibody syndrome), prolonged pregnancy, and/or impaired placental perfusion with subsequent fetal hypoxia and growth restriction. If growth restriction is suspected, fetal measurements should be obtained, amniotic fluid index quantified, and a careful fetal anatomical survey should be performed. If growth is less than expected for gestational age, an umbilical artery systolic/diastolic ratio (S/ D) and a middle cerebral artery pulsatility index (PI) should be obtained. An elevated S/D ratio, absent or reversed end-diastolic umbilical artery flow (Fig. 3), and decreased middle cerebral artery pulsatility index (Fig. 4) are consistent with increased placental resistance and fetal brain-sparing in the growth restricted fetus.

Oligohydramnios occasionally occurs in otherwise normal pregnancies, and in this situation does not appear to increase fetal risk (though at present only limited outcome data are available regarding this group of patients). Women at term undergoing labor induction for isolated oligohydramnios and women in spontaneous labor with normal amniotic fluid volumes have similar neonatal outcomes, but the relative risk for cesarean is increased 2.4-fold in women undergoing labor induction. ${ }^{23}$ Additionally, up to $50 \%$ of women diagnosed with decreased AFI will normalize amniotic fluid measurements within days. ${ }^{24}$ Though the significance of isolated oligohydramnios is unclear, labor is frequently induced, particularly at term. This may place women at risk for increased morbidity without improving fetal and neonatal outcomes. Further research is warranted to determine the optimal antepartum management of isolated oligohydramnios.

\section{HYDRATION}

Maternal hydration status can significantly affect AFI. Oral maternal hydration with 2 liters of water increases the AFI by $30 \%$ in women diagnosed with decreased AFI. ${ }^{25}$ Hypotonic

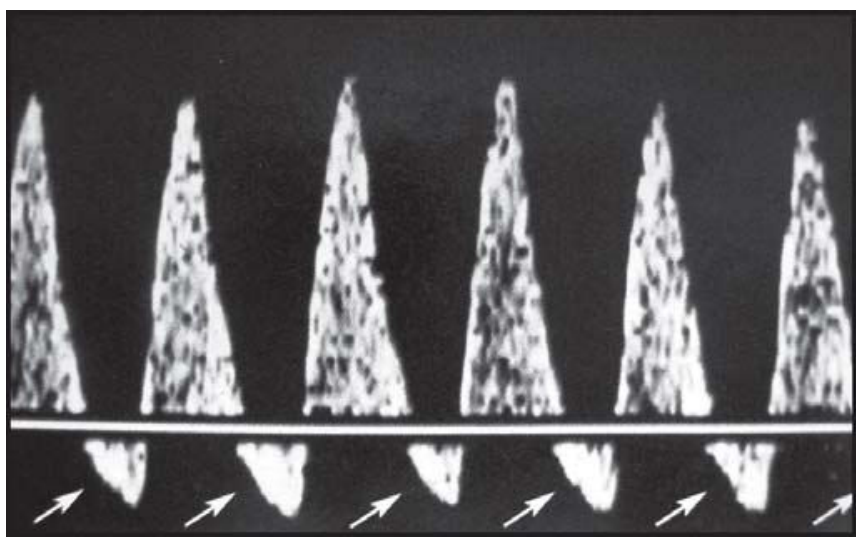

Fig. 3: Reversal of umbilical artery end diastolic flow, consistent with uteroplacental insufficiency

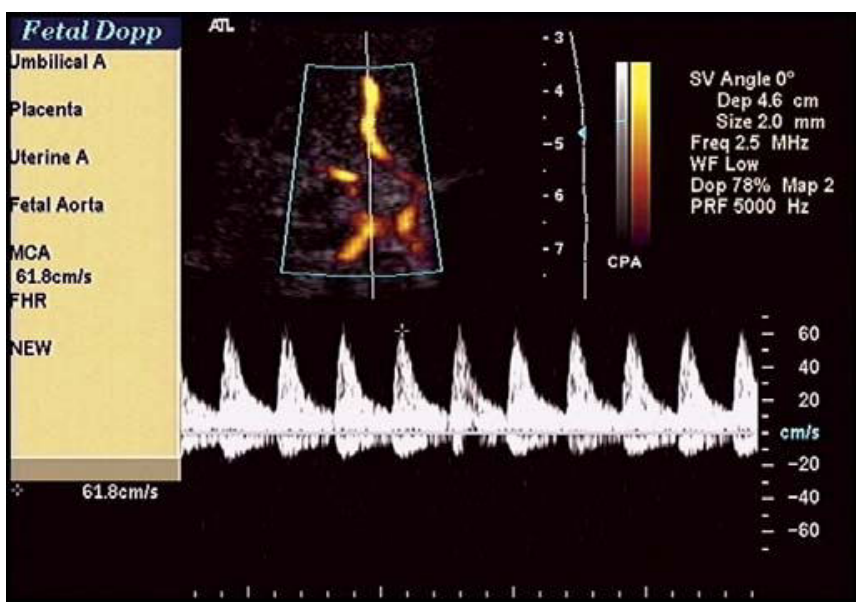

Fig. 4: Measurement of middle cerebral artery peak systolic velocity

intravenous fluid has also been shown to increase AFI in women with oligohydramnios. ${ }^{26}$ Of interest, isotonic intravenous fluid does not affect amniotic fluid volume, suggesting maternal plasma osmotic changes (not intravascular volume) affects AFI. Thus, maternal plasma osmolality could potentially be altered to treat oligohydramnios. Desmopressin (DDAVP) has been used in conjunction with water ingestion in research protocols with some success. ${ }^{2}$ DDAVP minimizes maternal fluid loss resulting in maternal and fetal hypo-osmolality. Thus fetal urine output and amniotic fluid volumes increase. Further studies are warranted to determine efficacy of treatment, and to address concerns regarding maternal and fetal safety.

\section{ROLE OF AMNIOINFUSION}

During labor, restoring the amniotic fluid volume with saline amnioinfusion has been advocated as a technique to decrease severe variable decelerations, operative deliveries for fetal distress, meconium passage and to increase umbilical artery 
blood $\mathrm{pH}$ values. It has also been recommended in the presence of meconium-stained amniotic fluid as a method of reducing the incidence of meconium aspiration syndrome. Amnioinfusion decreases the severity of repetitive variable decelerations and can lower the incidence of cesarean section for fetal distress. ${ }^{27}$ Though the procedure is considered safe, rare side effects include an increased rate of chorioamnionitis and uterine overdistension. Though amnioinfusion has demonstrated benefit in the presence of repetitive variable decelerations, controversy exists regarding the benefit of prophylactic amnioinfusion for oligohydramnios in the absence of decelerations. Early studies compared women receiving prophylactic amnioinfusion to women receiving no therapy, regardless of the presence or absence of decelerations. ${ }^{28,29}$ These studies showed prophylactic amnioinfusion decreased operative interventions for fetal distress and decreased the rate of cesarean birth. Subsequent studies compared women receiving prophylactic amnioinfusion for oligohydramnios to women receiving therapeutic amnioinfusion for fetal heart rate variable decelerations. ${ }^{30,31}$ These studies failed to show a benefit of prophylactic treatment. Thus, there is no clear evidence prophylactic amnioinfusion for oligohydramnios is of benefit. Additionally, though initial studies appeared to suggest amnioinfusion reduces the incidence of meconium aspiration syndrome, a recent large, multicenter, randomized trial in women with thick meconium-stained amniotic fluid failed to show any benefit to saline amnioinfusion. ${ }^{32}$ Therefore, prophylactic amnioinfusion for meconium-stained amniotic fluid is not recommended. ${ }^{33}$ During labor, amnioinfusion is indicated for repetitive variable decelerations regardless of presence or absence of meconium.

\section{REFERENCES}

1. Chamberlain PS, Manning FA, Morrison I, Harman C, Lange I. Ultrasound evaluation of amniotic fluid volume: the relationship of marginal and decreased amniotic fluid volumes to perinatal outcome. Am J Ostet Gynecol 1984;150:245-9.

2. Ross MG, Brace RA, and the NIH workshop participants. National Institute of Child Health and Developmental Conference summary: amniotic fluid biology — basic and clinical aspects. J Mat Fetal Med 2001;10:2-19.

3. Gilbert WM, Brace RA. The missing link in amniotic fluid volume regulation: intramembranous absorption. Obstet Gynecol 1989;74:748-54.

4. Gilbert WM, Eby-Wilkens E, Tarantal AF. The missing link in rhesus monkey amniotic fluid volume regulation: intramembranous absorption. Obstet Gynecol 1997;89:462-5.

5. Faber JJ, Anderson DF. Regulatory response of intramembranous absorption of amniotic fluid to infusion of exogenous fluid in sheep. Am J Physiol 1999;277:R236-42.

6. Brace RA, Wolf EJ. Normal amniotic fluid volume changes throughout pregnancy. Am J Obstet Gynecol 1989;161:382-8.
7. Moore TR, Cayle JE. The amniotic fluid index in normal human pregnancy. Am J Obstet Gynecol 1990;162:1168-73.

8. Manning FA, Platt LD, Sipos L. Antepartum fetal evaluation: development of a fetal biophysical profile. Am J Obstet Gynecol 1980;136:787-95.

9. Chamberlain PF, Manning FA, Morrison I, Harman CR, Lange IR. Ultrasound evaluation of amniotic fluid volume. Am J Obstet Gynecol 1984;150:245-54.

10. Mercer LJ, Brown LG, Petres RE, Messer RH. A survey of pregnancies complicated by decreased amniotic fluid. Am J Obstet Gynecol 1984;149:355-61.

11. Phelan JP, Smith CV, Small M. Amniotic fluid volume assessment with the four quadrant technique at 36-42 weeks' gestation. J Repord Med 1987;32:540-2.

12. Moore TR. Superiority of the four-quadrant sum over the single deepest pocket technique in ultrasonographic identification of abnormal amniotic fluid volumes. Am J Obstet Gynecol 1990;163:762-7.

13. Magann EF, Perry KG, Chauhan SP, Anfranger PJ, Whitworth NS, Morrison JC. The accuracy of ultrasound evaluation of amniotic fluid volume in singleton pregnancies: the effect of operator experience and ultrasound interpretive technique. J Clin Ultrasound 1997;25:249-53.

14. Carlson DE, Platt LD, Medearis AL, Hornestein J. Quantifiable polyhydramnios: diagnosis and management. Obstet Gynecol 1990;75:989-91.

15. Hill LM, Breckle R, Thomas ML, Fries JK. Polyhydramnios: ultrasonically detected prevalence and neonatal outcome. Obstet Gynecol 1987;69:21-5.

16. Maymon E, Ghezzi F, Shoham-Vardi I, Franchi M, Silberstein T, Wiznitzer A, Mazor M. Isolated hydramnios at term gestation and the occurrence of peripartum complications. Eur J Obstet Gynecol Reprod Biol 1998;77:157-61.

17. Kirshon B, Mari G, Moise KJ. Indomethacin therapy in the treatment of symptomatic polyhydramnios. Obstet Gynecol 1990; 75:202-5.

18. Kirshon B, Moise KJ, Wasserstrum N, OU CN, Huhta JC. Influence of short-term indomethacin therapy on fetal urine output. Obstet Gynecol 1988;72:51-3.

19. Walker MPR, Moore TR, Brace RA. Indomethacin and argininevasopressin interaction in the fetal kidney: a mechanism of oliguria. Am J Obstet Gynecol 1994;171:1234-41.

20. Moise KJ Jr, Huhra JC, Sharif DS, Ou CN, Kirshon B, Wasserstrum N, Cano L. Indomethacin in the treatment of premature labor: effects on the fetal ductus arteriosus. N Engl J Med 1988;319:327-31.

21. Brace RA, Wolf EJ. Normal amniotic fluid volume changes throughout pregnancy. Am J Obstet Gynecol 1989;161:382-8.

22. Chamberlain P. Amniotic fluid volume: ultrasound assessment and clinical significance. Semin Perinatol 1985;9:163-7.

23. Conway DL, Adkins WB, Schroeder B, Langer O. Isolated oligohydramnios in the term pregnancy: is it a clinical entity? J Matern Fetal med 1998;7:197-200.

24. Garmel SH, Chelmow D, Sha SJ, Roan J, D'Alton ME. Oligohydramnios and the appropriately grown fetus. Am J Perinatol 1997; 14:359-3. 
25. Kilpatrick SJ, Safford KL, Pomeroy T, Hoedt L, Scheerer L, Laros RK. Maternal hydration increases amniotic fluid index. Obstet Gynecol 1991;78:1098-102.

26. Flack NJ, Sepulveda W, Bower S, Fisk NM. Acute maternal hydration in third-trimester oligohydramnios: effects on amniotic fluid volume, uteroplacental perfusion, and fetal blood flow and urine output. Am J Obstet Gynecol 1995;173:1186-91.

27. Miyasaki FS, Nevarez F. Saline amnioinfusion for relief of repetitive variable decelerations: a prospective randomized study. Am J Obstet Gynecol 1985;153:301-6.

28. Schrimmer DB, Macri CJ, Paul RH. Prophylactic amnioinfusion as a treatment for oligohydramnios in labor patients: a prospective randomized trial. Am J Obstet Gynecol 1991;165:972-5.

29. Strong TH, Hetzler G, Sarno AP, Paul RH. Prophylactic intrapartum amnioinfusion: a randomized clinical trial. Am J Obstet Gynecol 1990;163:1370-5.
30. MacGregor SN, Banzhaf WC, Silver RK, Depp R. A prospective randomized evaluation of intrapartum amnioinfusion. J Reprod Med 1991;36:69-73.

31. Ogundipe OA, Spong CY, Ross MG. Prophylactic amnioinfusion for oligohydramnios: a reevaluation. Obstet Gynecol 1994;84:544-8.

32. Fraser WD, Hofneyr J, Lede R, Faron G, Alexander S, Goffinet F, Ohlsson A, Goulet C, Turcot-Lamay L, Prendeville W, Marcous S, Laperriere L, Roy C, Petroux S, Xu HR, Wei B. Amnioinfusion trial group. Amnioinfusion for the prevention of the meconium aspiration syndrome. N Engl J Med 2005;353:90917.

33. ACOG committee opinion. Amnioinfusion does not prevent meconium aspiration syndrome. Obstet Gynecol 2006; 108(4):1053-5. 